\title{
Iterative Algorithms for Deblurring of Images in Case of Electrical Capacitance Tomography
}

\author{
Nasif R. Jaffri $\mathbb{D}^{1},{ }^{1,2}$ Ahmad Almogren $\left(\mathbb{D},{ }^{3}\right.$ Aftab Ahmad $\left(\mathbb{D},{ }^{2}\right.$ Usama Abrar $\left(\mathbb{D},{ }^{1,2}\right.$ \\ Ayman Radwan ${ }^{D}, 4$ and Farzan A. Khan iD ${ }^{5}$ \\ ${ }^{1}$ School of Control and Computer Engineering, North China Electric Power University Beijing, Beijing 102206, China \\ ${ }^{2}$ Department of Electrical Engineering, NFC-Institute of Engineering and Fertilizer Research Faisalabad, Faisalabad, Pakistan \\ ${ }^{3}$ Department of Computer Science, College of Computer and Information Sciences, King Saud University, \\ Riyadh 11633, Saudi Arabia \\ ${ }^{4}$ Instituto de Telecomunicações and Universidade de Aveiro, Aveiro, Portugal \\ ${ }^{5}$ Office of Global Education, New York University, Abu Dhabi 129188, UAE
}

Correspondence should be addressed to Aftab Ahmad; aftabahmadrao@gmail.com

Received 4 May 2021; Revised 3 July 2021; Accepted 16 July 2021; Published 11 August 2021

Academic Editor: Jakub Grabski

Copyright (C) 2021 Nasif R. Jaffri et al. This is an open access article distributed under the Creative Commons Attribution License, which permits unrestricted use, distribution, and reproduction in any medium, provided the original work is properly cited.

Electrical capacitance tomography (ECT) has been used to measure flow by applying gas-solid flow in coal gasification, pharmaceutical, and other industries. ECT is also used for creating images of physically confined objects. The data collected by the acquisition system to produce images undergo blurring because of ambient conditions and the electronic circuitry used. This research includes the principle of ECT techniques for deblurring images that were created during measurement. The data recorded by the said acquisition system ascends a large number of linear equations. This system of equations is sparse and illconditioned and hence is ill-posed in nature. A variety of reconstruction algorithms with many pros and cons are available to deal with ill-posed problems. Large-scale systems of linear equations resulting during image deblurring problems are solved using iterative regularization algorithms. The conjugate gradient algorithm for least-squares problems (CGLS), least-squares QR factorization (LSQR), and the modified residual norm steepest descent (MRNSD) algorithm are the famous variations of iterative algorithms. These algorithms exhibit a semiconvergence behavior; that is, the computed solution quality first improves and then reduces as the error norm decreases and later increases with each iteration. In this work, soft thresholding has been used for image deblurring problems to tackle the semiconvergence issues. Numerical test problems were executed to indicate the efficacy of the suggested algorithms with criteria for optimal stopping iterations. Results show marginal improvement compared to the traditional iterative algorithms (CGLS, LSQR, and MRNSD) for resolving semiconvergence behavior and image restoration.

\section{Introduction}

Industrial tomography offers a prospect to measure and visualize the physically constrained industry operations [1]. Industrial tomography is divided into two categories: (i) hard field and (ii) soft field. This categorization of tomography is according to the measurement principles. X-ray, positron emission particle tracking (PEPT), $\gamma$-ray tomography, and so forth fall under the group of hard-field tomography. The latter group, named soft field, comprises microwave tomography (MWT), electrical impedance tomography (EIT), and electrical capacitance tomography (ECT) $[2,3]$. ECT has many benefits when used in a gas-solid flow, such as being less prone to signal loss and being easier to automate. As coal combustion processes typically use nonconductive materials, such as powdered coal, the circulating fluidized bed (CFB) [4] processes use ECT because it is the most acceptable option for these processes. ECT is applicable in rough ambient conditions where its tolerance to high temperature and pressure is required. It is less priced, fast, safe, and easy to use. In particular, ECT can offer extra data on the gas-solid flow hydrodynamics [5], for example, 
flow transition from bubbling to slowing flux for scaling the CFB process from the laboratory scale to the production scale with diverse riser structures and cyclones. ECT is commonly used in many other imaging modalities for visualization besides magnetic resonance imaging (MRI) [6] and X-ray tomography [7].

Since the invention of tomographic systems, the foremost goal of the system has been to detect/sense the complex data followed by its meaningful interpretation. This interpretation helps estimate qualitative and quantitative information about the behavior of fluids moving within the process [8]. These measurements may be taken across pipes, vessels, storage tanks, or reactors. The origin of this extension of science is attached to medical science, where different tomographic techniques are applied to get the data of static parts from the human body [9]. Later, the static values of measurand using these tomographic techniques were applied.

Semiconvergence is the main problem in iterative algorithms, like any other algorithm. This work spotlights the deblurring of image restored from the received data in industrial tomography effectively to handle semiconvergence [10]. The iterative technique enhances image quality with minimal error and converges at the optimal point by implementing proposed algorithms using Krylov solvers for linear systems [11].

The measurement from ECT (or any tomography) leads towards a system of large sparse linear equations [12]. Since these systems are ill-posed in nature, this means that they can converge to more than one solution. In order to come across a unique solution, a variety of algorithms have been deployed. The iterative algorithms are the foremost choice in such scenarios [13]. Furthermore, the reconstructed image is subject to blurring [14] and speckling [15] because of the environment and electric circuitry used. This work emphasized the removal of such noise during and after measurement.

When images are taken, it is normal for them to be blurred. Image deblurring is the method of restoring an approximate image by modeling it as the solution of the linear operator equation $[16,17]$.

$$
U x=Y, \quad U \varepsilon R^{M x N}, x \varepsilon R^{N},
$$

where $x$ is a vector of the actual image we aim to deblur. The matrix $U$ is ill-determined, referred to as the ill-posed problem, and $Y$ matrix is vector values at the receiving end [18]. This value of the right-hand side is generally obtained through measurement.

$\widehat{Y}$ is an error-free matrix, whereas $Y_{n}$ represents random noise added during measurement. Similarly, $x=\widehat{x}+x_{n}$; in this expression, $x_{n}$ is the error that deblurs the image, and we try to eliminate it.

Problems like these are cropped up in astronomy, imaging for the medical field, and geophysics. In general, discretization of an ill-posed continuous model produces equation (1). For example, discretization means that matrix $U$ could be illconditioned [12], or its data could be distorted, which could lead to inaccuracies in the approximate solution. Directly solving equation (1) without error results in an inaccurate and unstable approximate solution, so the equation must be regularized first. Many regularization technologies such as the Tikhonov regularization [19], truncated iterative algorithms [20] (e.g., steepest descent and conjugate gradient (CG) iterations), decomposition of truncated singular value (TSVD) [21], and hybrid methods [22] have been regularly studied and discussed in the literature.

It is critical to set the regularization parameter to an appropriate value while incorporating it with a regularization method. The fixed-point algorithm, generalized cross-validation (GCV), the weighted-GCV (W-GCV) [23], L-curve [24], and discrepancy principle [25] are suitable for Tikhonov regularization [19] as a technique for parameter choice. There are some advantages and disadvantages in the parameter selection methods [26]. Choosing an optimal regularization parameter is a complex task. Tikhonov regularization may not be a suitable alternative to iterative algorithms like CG and steepest descent, which use iterations to speed up convergence. Only through matrix-vector multiplication with $U$ or $U^{T}$ do they have access to the coefficient matrix $U$. The iterative regularization algorithms can be tricky to apply to solve the linear system $U x=Y$ due to the semiconvergence behavior [10]. The phenomenon under discussion is that the initial iterations of the algorithm lead to regularization solutions, and, after a small number of iterations, the estimated results converge to some further undesirable vector referred to as the first-order regularization. This unwanted vector is corrupted by errors and thus an inaccurate representation. Another way of saying this is that, in essence, an erroneous calculation of the stopping iteration would yield a suboptimal solution, which necessitates choosing a stopping point for the iterations. Techniques such as the difference theory and the L-curve may be used for the selection of such an appropriate termination [24]. Still, as with the Tikhonov regularization, it is also nontrivial [19].

Reducing the complexity of finding the termination iteration number of iterative regularization algorithms is partly achieved by hybrid approaches. Combining an iterative Lanczos bidiagonalization algorithm $[11,27]$ with a regularization algorithm such as Tikhonov regularization and TSVD allows for a hybrid approach that uses an iterative Lanczos algorithm tandem with a regularization algorithm such as Tikhonov regularization and TSVD at each iteration. Therefore, regularization is accomplished by filtering Lanczos bidiagonalization and by choosing a parameter for regularization on each iteration [27]. W-GCV has recently been researched in the sense of the Lanczos-Tikhonov hybrid method. This Lanczos-Tikhonov hybrid method will reduce the iteration number's impact on the solution. But the semiconvergence property is characterized by its proceeding iteration. In combination with Tikhonov regularization in the generated Krylov subspace the GKB algorithm's regularization parameters for the partial Golub-Kahan iteration are selected [11]. GKB solutions, in some cases, are more accurate than W-Lanczos-Tikhonov GCV's. The two strategies are nevertheless comparatively successful in some situations.

Three iterative algorithms that are built on the iterative regularization are proposed in this work and are acted as image deblurring algorithms and soft thresholding. Note that $\widehat{Y}$ is noise in right-hand and this noise propagates with each iteration. The propagation of the noise causes 
semi-convergence behavior in iterative algorithms such as CGLS, LSQR, and MRNSD [10, 28]. Combining iterative regularization algorithms and a noise lessening method like soft thresholding at every iteration will allow us to overcome the semiconvergence behavior of iterative regularization. A new algorithm has one part where soft thresholding is used in conjunction with CGLS and LSQR and another part where soft thresholding is used in conjunction with MRNSD. Because of these algorithms, the resulting models are robust and highly efficient in practical applications. Our analysis shows that MRNSD and soft thresholding are both convergent algorithms. The computational tests demonstrate that the suggested algorithms resolve semiconvergence and restoration behaviors. The results of CGLS, LSQR, and MRNSD are only marginally better than their optimal iterations [29].

Tomographic technology is done by collecting data on the boundary of an area like a process vessel or pipeline of sensor signals. This process reveals information on component characteristics and distribution within the sensing field $[3,30]$. Most tomography techniques concern the creation of a cross-sectional image by abstracting details. For example, a parallel set of sensors (Figure 1) can be used to analyze the projections of suitable radiation in the subject as expected to be a circular cross section in their sensing area $[31,32]$. To analyze the whole cross section, other projections must be obtained by rotating the object to the direction of the sensor field or, ideally, by rotating the sensors around the subject. Such a technique may not always be suitable because it may be impractical to rotate the subject or the sensors physically. It may take too long for the assembly to rotate relative to changes in the subject under review [6].

ECT has a basic principle: Capacitance measurement by a sensor having several electrodes surrounding a nonconductive industrial procedure, such as a gas-solid flow, is performed to measure the process's impedance. The region that measures the capacitance of different materials has been partitioned into a higher number of virtual pixels, which yields the visualization of the cross-sectional distribution of object(s) under study. Image reconstruction algorithms are then used to recreate the image from the calculated capacitance data and the result. An ECT system is made up of three different parts: sensors, data acquisition boxes, and computers work the image restoration algorithms as shown in Figure 2.

Generally, an ECT sensor is typically fitted with the variable number of electrodes uniformly arranged around a pipeline or vessel, for example, in a gas-solid CFB. The electrode and a grounded screen have an isolating sheet among them. A picture of the setup used is shown in Figure 3. The specification and data acquisition are provided in [33].

Reconstruction of an image in ECT is usually an ill-posed inverse problem, meaning that inverse problems can converge to more than one solution. Hence unique solution needs vigorous calculations to find. Some years ago, image reconstruction algorithms for ECT were tested [34]. There have since been several new or updated image reconstruction algorithms designed to overcome the ill-posed and ill-conditioned problem. Categories usually involve three classes. The first class of algorithms is the noniterative, and the second class is the iterative. Lastly, there is a third class that implements direct

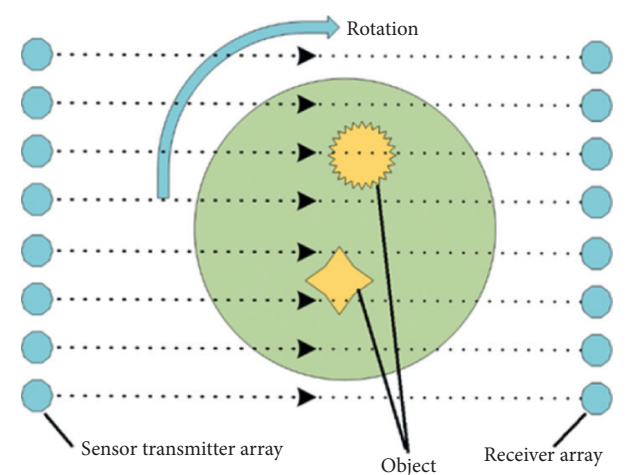

FIgURE 1: Use of projections to generate an image.

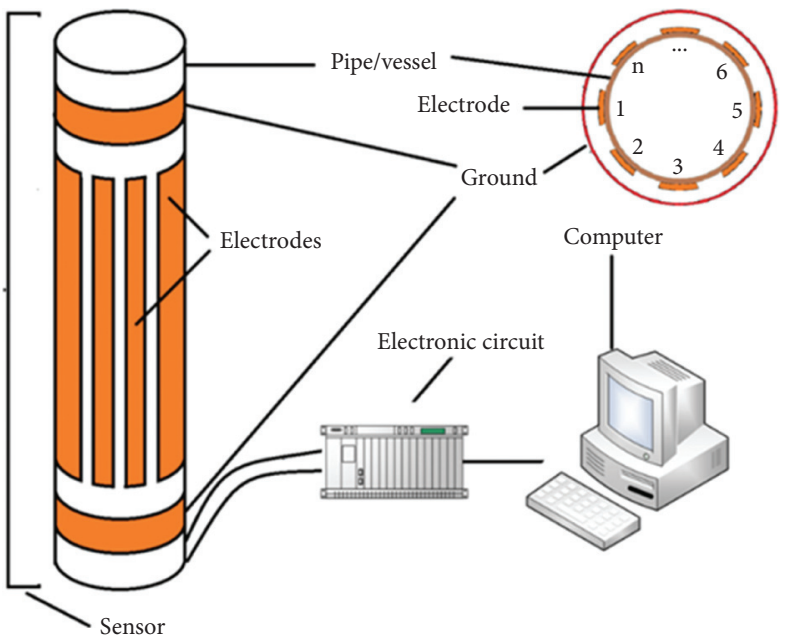

Figure 2: Layout of our ECT system used.

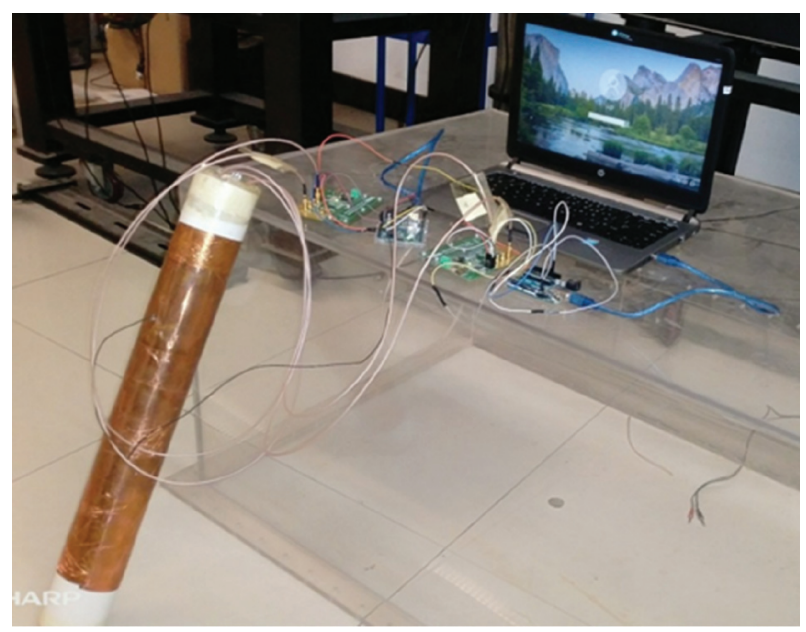

FIgURE 3: ECT sensor used for data acquisition.

mapping. The iterative image reconstruction technique aims to lessen the capacitance error while also enhancing image quality.

There are several popular algorithms for solid distribution reconstruction in a fluidized bed. Among these, the Landweber iteration and the linear back projection (LBP) are the most famous [20, 35]. LBP is fast and reliable. 
Nevertheless, it does abysmally for complex distribution, that is, fluidized bed crown-like solids distribution. It is possible to apply Landweber iteration to improve the image quality. All reconstruction algorithms are transform-based or iterative. Abel inversion is for spherical or axial symmetry [21]. In case of filter back projection algorithm (FBPA), intensive projections are needed for desired measurements. However, maximum likelihood expectation maximization (ML-EM) has multiple maxima with no guarantee of global maxima [23]. Algebraic Reconstruction Techniques (ART) is good as it has few projections required for squared problems but low resolution. The steepest Descent problems used here deal with nonsquare problems. This noise data, being correlated. There exist various algorithms to dispense these spots. Some of the PDE methods are quick but lose significant picture data during despeckling. Song et al. [36] investigated the effects of the projected angle and number of rays on the temperature reconstruction, and the results were computed for both fan beam and parallel beam perspective. It was observed that poor reconstruction usually appears on the corner and the center of the reconstruction area. However, no software technique was used to enhance the image.

One of the assignments is to device an ECT sensor. In the ECT sensor design, the different factors include temperature, strain, being nonintrusive and noninvasive, and a diverse potent range [8]. To obtain gas-solid fluidized beds of various dimensions and structures and to investigate gassolid fluidized beds of various dimensions and structures, ECT sensors have to be specially built and calibrated to attain better-quality images. In the commercial implementation of ECT in CFBs, effective sensor construction and optimized design are key factors. The image quality is defined by the signal-to-noise ratio (SNR) for ECT. SNR is a feature of both the acquisition system used and the sensor layout [31]. The second problem is the AC-based ECT system's operation parameter configuration.

Additionally, it will be discussed later regarding the SNR evaluation for various structures and diverse measurements of ECT sensors. Additionally, as the sensitivity in the center of an ECT sensor decreases with the rise in the diameter of the ECT sensor, poor image quality is obtained with a largescale visualization problem [37]. The capacitive measurement by the ECT system of Figure 3 induction of Gaussian noise is used as input data.

\section{Methodology}

The data for noise applied during the analysis is correlated, making it easier to delete. Many different approaches are used to remove the speckles. Some of the PDE methods are quick but lose significant picture data during despeckling proceedings. For this work, the variation of iterative algorithms from CGLS, LSQR, and MRNSD was used to eliminate blurring, hence visualizing a pattern of objects confined within the area under study [10]. The measured data were collected with the help of electrical capacitance tomographic system. Clear and blurred images were convolued to carry out the deblur process. By doing this, a system of ill-posed linear equations was evolved, as represented in equation (1). Finally, equation (2) represents the matrix of equation (1).

$$
\left[\begin{array}{ccccc}
U_{12} & U_{13} & \cdots & \cdots & U_{1 N} \\
U_{21} & U_{21} & \cdots & \cdots & U_{2 N} \\
\vdots & \vdots & \vdots & \vdots & \vdots \\
U_{M 1} & U_{M 1} & \cdots & \cdots & U_{M N}
\end{array}\right] \times\left[\begin{array}{c}
x_{1} \\
x_{2} \\
\vdots \\
\vdots \\
x_{N}
\end{array}\right]=\left[\begin{array}{c}
Y_{1} \\
Y_{2} \\
\vdots \\
Y_{M}
\end{array}\right] .
$$

In each iteration, there is the propagation of noise on the right side of $Y$ because of $Y_{n}$. Because matrix $U$ is ill-conditioned and propagates noise with iteration, the quality of the image first improves and then declines. The outcome results in iterative algorithms like CGLS, LSQR, and MRNSD being moderately convergent. This work was formulated on the base of iterative algorithm with a blend of image deblurring soft-thresholding. Additionally, an appropriate stopping criterion for the algorithm is developed to halt the convergence at optimal iteration. According to [10], the soft-thresholding operator $S_{\mu}$ is represented in equation (3) and $k$ has variable values up to grid point $N$.

$$
\left(S_{\mu}\left(x_{k}\right)\right)_{k}=\operatorname{sgn}\left(x_{k}\right) \cdot \max \left\{\left|x_{k}\right|-\frac{\mu}{2}, 0\right\}, \quad k=1,2, \ldots, N, x \varepsilon R^{N} .
$$

The product of the sparsity of the large matrix $U$ and the diagonal is a measure of the proportion of nonzero elements in most of the problems. There is more than one solution to ill-posed issues to keep the solution of matrix $x$ with the minimum $U x-Y$; it is an element of the error to equation (4). By using rank, the error solution is taken to the lowest possible value. Parabolic-shaped quadratic equation (5) is assumed, and the gradient is determined as shown in equation (6).

$$
\begin{aligned}
U^{T} U x & =U^{T} Y, \\
f(x) & =x^{T}\left(U^{T} U\right)-2\left(U^{T} Y\right)^{T} x, \\
f^{\prime}(x) & =2 U^{T} U x-2 U^{T} Y .
\end{aligned}
$$

To determine the solution of an ill-posed matrix, equation (4) is sufficient. Generally, the preferred method is to describe a point $x_{0}$ and then find the solution as the function approaches zero [38]. The next iteration is set to be at the next stage, $x_{i+1}$, that function reached $x_{i}$. Once the function gradient is equal or approximately equal to zero, the function will converge.

The residual error in equation (1) is (estimated) explained as $e_{i}=Y-U x_{i}$. What you have discovered explains how close $U x_{i}$ and $Y$ are to each other. Similarly, $q_{i}=$ $U^{T} Y-U^{T} U x_{i}$ shows residual equation error (4). Furthermore, $U^{T} Y$ and $U^{T} U x_{i}$ are at a distance from each other. This separation is defined by the following equation:

$$
x_{i+1}=x_{i}+\eta_{i} q_{i}
$$


As per requirement, $q_{i}$ and $q_{i+1}$ are made orthogonal to each other. This pattern of recurrence for the steepest descent is described by equation (9), while $\eta_{i}$ is shown by equation (8).

$$
\begin{gathered}
\eta_{i}=\frac{q_{i}^{T} q_{i}}{q_{i}^{T} U^{T} U q_{i}}, \\
e_{i+1}=e_{i}-\eta_{i} U q_{i} .
\end{gathered}
$$

The steepest descent algorithm terminates at optimal iteration to prevent the semi-convergence behavior, equation (10) and equation (11) [10] were used. Equation (10) computes maxima of $Y$, and (11) is a search to ensure that $e_{i}$ is small or less than zero.

$$
\begin{aligned}
k(Y) & =\frac{\sigma_{\max }(Y)}{\sigma_{\min }(Y)}, \\
\left\|e_{i}\right\| & \leq\left(\frac{k\left(U^{T} U\right)-1}{k\left(U^{T} U\right)+1}\right) .
\end{aligned}
$$

2.1. The Method of Conjugate Gradients for Least Squares (CGLS). For this CGLS, there is only one change to steepest descent, and it pursuits in the direction of the specified userdefined point $p_{i}$ instead of $q_{i}$ [29]. This $p_{i}$ relies on variable $\gamma \cdot \gamma_{i}$ will be given for soft thresholding, and the equation is given as follows:

$$
\gamma_{i}=S^{T} S_{i}
$$
1

The following is the algorithm for CGLS [10]. Algorithm

2.2. The Method of the Minimal Residual Norm of Steepest Descent (MRNSD). The CGLS algorithm [10] is fast, and the Krylov solver is un restricted. The unconstrained system aims to protect unaltered positivity [39]. Another way to do this is to add a constraint to the equation, if we are going to regularize the least-square functions [21].

$$
J(x)=\frac{1}{2}\left\|U x-Y_{2}^{2}\right\| .
$$

Now, parametrizing $x=k^{z}$ and taking the transform, we get

$$
J(x)=\frac{1}{2}\left\|U x-Y_{2}^{2}\right\| .
$$

Applying the chain rule, it is concluded that

$$
\begin{aligned}
\operatorname{grad}_{z} \bar{J}(z) & =X_{\operatorname{grad}_{x} J(f)=X U^{T}(U x-Y),} \\
X & =\operatorname{diag}(x) .
\end{aligned}
$$

Equation (14) is taken into account for the MRNSD algorithm [10] that differs from CGLS.

The following is the algorithm for MRNSD [10]. Algorithm 2

2.3. The Method for Least Square for QR-Factorization (LSQR). LSQR is based on Lanczoss bidiagonalization algorithm [11]. The trajectories of both LSQR and MRNSD are similar as they follow similar trajectories in kind. The LSQR derivation is nevertheless not as simple as MRNSD [28].

$$
\begin{aligned}
U V_{i} & =W_{i+1} Y_{i}, \\
U^{T} W_{i+1} & =V_{i} Y_{i}^{T}+\eta_{i+1} v_{i+1} g_{i+1}^{T} .
\end{aligned}
$$

$g_{i+1}=(0, \ldots, 0,1)^{T} \varepsilon R^{i+1}, \quad V_{i}=\left(v_{1}, v_{2}, \ldots, v_{i}\right), \quad$ and $W_{i+1}=\left(u_{1}, u_{2}, \ldots, u_{i+1}\right)$ are orthonormal columns. The $(i+$ 1) $\times i$ lower bidiagonal matrix is denoted as

$$
W_{i+1}^{T} U V_{i}=Y_{i}=\left[\begin{array}{cccc}
\eta_{1} & & & \\
\gamma_{2} & \eta_{2} & & \\
& \gamma_{3} & \ddots & \\
& & \ddots & \eta_{i} \\
& & & \gamma_{i+1}
\end{array}\right] \in R^{(i+1) \times i} .
$$

The following is the algorithm for LSQR [40]. Algorithm 3

\section{Results and Discussion}

Simulations were performed in MATLAB for 100 iterations to show image reconstruction implementation. Iterations used CGLS, LSQR, and MRNSD iterative deblurring algorithms to fulfill the objectives.

MRNSD applies only to least-square weighted problems. The only difference between CGLS and MRNSD is that CGLS does not require being nonnegative [7]. CGLS, LQR, and MRNSD are algorithms for solving least-squares problems. The first two solvers are unconstrained, while the third is constrained. Therefore, the truth value is not preserved by the first solver. Given the problem in Figure 4, the algorithms were run on a computer with 10th Generation Intel ${ }^{\circledR}$ Core $^{\mathrm{TM}}$ i7-10510U (8 MB Cache, four-core, $1.8 \mathrm{GHz}$ to $4.9 \mathrm{GHz}$ ) and $32 \mathrm{~GB}, 2 \times 16 \mathrm{~GB}$, DDR4 RAM. A 32-bit index image was generated by adding 6\% Gaussian noise to a $512 \times 512$ image, as can be seen in Figure 5 .

The $6 \%$ Gaussian white noise is used to blur the original $256 \times 256$ image shown in Figure 4. The blurred images and the restored images using three algorithms are seen in Figure 6 . Figures 5 and 6 show that the images restored by the MRNSD algorithm have less noise and are smoother than CGLS and LSQR images. MRNSD is robust but not as robust as the other two algorithms and converges at a higher number of iterations.

Figure 7 shows the error graph for the CGLS norm versus the iterations on the $x$-axis. The semiconvergence behavior is very evident. The error decreases till the 12th iteration, and, after that, it shoots up. The iteration is marked with a red circle to represent where the algorithms stopped.

Similarly, Figure 8 represents the LSQR error-norm graph. The iteration with the lowest error for this algorithm is the 29th iteration, and, as expected, the error started to rise. This section may be divided into subheadings. It should provide a concise and precise description of the experimental results and their interpretation, as well as the experimental conclusions that can be drawn. 
Given: Matrix $U$, initial guess $x_{0}$, Right-hand side $Y$ and soft-thresholding parameter $\mu>0$.

$e_{0}=Y-U x_{0}$

$q_{0}=U^{T} e_{0}$

$p_{0}=q_{0}$

for $i=1,2,3, \ldots$, do

$p_{i}=U q_{i}$

$\eta_{i}=\gamma_{i} / p^{T} p$

$U_{\text {old }}=U$

$U=U_{\text {old }}+\eta_{i} p_{i}$

$e_{i+1}=S_{\mu}\left(e_{i}-\eta_{i} U p_{i}\right)$

$S_{i}=U^{T} e_{i}$

$\gamma_{i}=S^{T} S_{i}$

end

Algorithm 1: Algorithm for CGLS.

Given: Ill-posed Matrix $U$, initial guess $x_{0}$, Right-hand side $Y$

$x_{i}=x_{0}$

$S_{0}=U^{T}\left(Y-U x_{0}\right)$

$X_{0}=\operatorname{diag}\left(x_{0}\right)$

$\gamma_{0}=S^{T} X_{0} S_{0}$

for $i=1,2,3, \ldots$, do

$q_{i}=X_{i} s_{i}$

$p_{i}=U q_{i}$

$\eta_{i}=\min \left(\left(\gamma / p^{T} p\right), \min _{q_{i}}<0\left(-\left(Y_{i} / q_{i}\right)\right)\right)$

$Y_{i}=Y_{i-1}+\eta_{i} q_{i}$

$X_{i}=\operatorname{diag}\left(x_{i}\right)$

$z_{i}=U^{T} p_{i}$

$S_{i}=S_{i-1}+\eta_{i} z_{i}$

$\gamma_{i}=S^{T} X_{i} S_{i}$

end

Algorithm 2: Algorithm for MRNSD.

Select unit length vector $w_{1} \varepsilon R^{M}, \gamma_{1} v_{0}=0$, and $\eta_{n+1} v_{n+1}=0$

for $j=1,2,3, \ldots, i$, do

$p=U^{T} w_{j}-\gamma_{j} v_{j}-1$

$\eta_{j}=\|p\| ; v_{j}=\left(p / \eta_{j}\right)$

$e=U v_{j}-\eta_{1} w_{j}$

$\gamma_{j+1}=\|e\| ; w_{j+1}=\left(e / \gamma_{j+1}\right)$

end

Algorithm 3: Algorithm for LSQR.

The MRNSD graph that is shown in Figure 9 shows semiconvergence. But the semiconvergence in MRNSD is not as vibrant as those in the other two algorithms, and the error line is a bit smooth.

Table 1 provides convergence error histories of all algorithms. For the test image, CGLS again displays a clear semiconvergence behavior. The symbol "*" is used with the error to represent the stopping iteration. For CGLS, it was the 12th iteration with an error of 0.186189 , whereas, for LSQR, it was 0.185546 , and it was 0.167680 for MRNSD. For CGLS and LSQR, these error values increase rapidly, but they are fractionally stable in the case of MRNSD. 


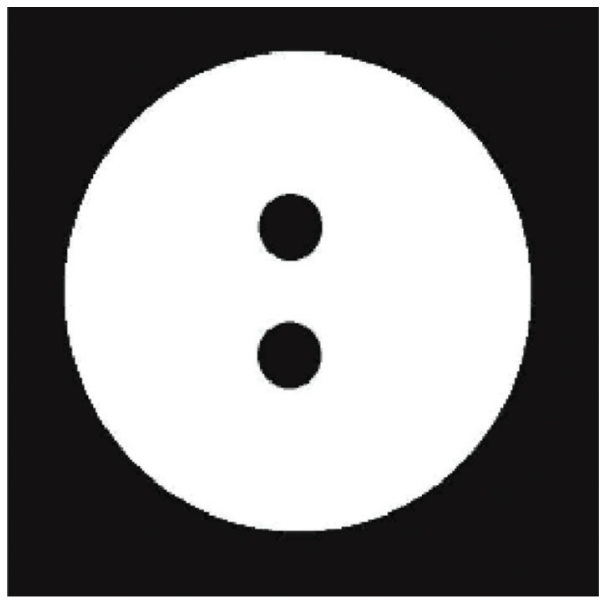

Figure 4: True image to be constructed.

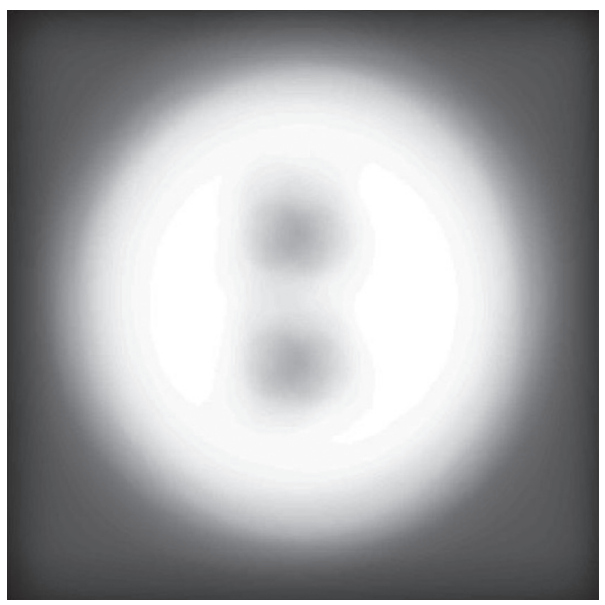

FIGURE 5: The image with induced noise.

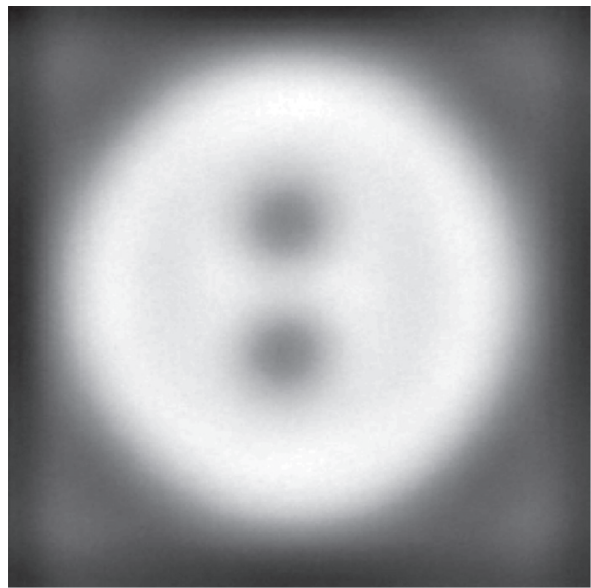

(a)

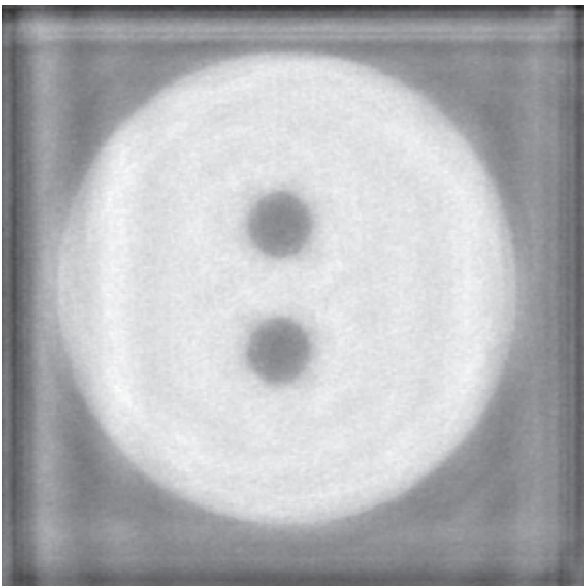

(b)

FIGURE 6: Continued. 


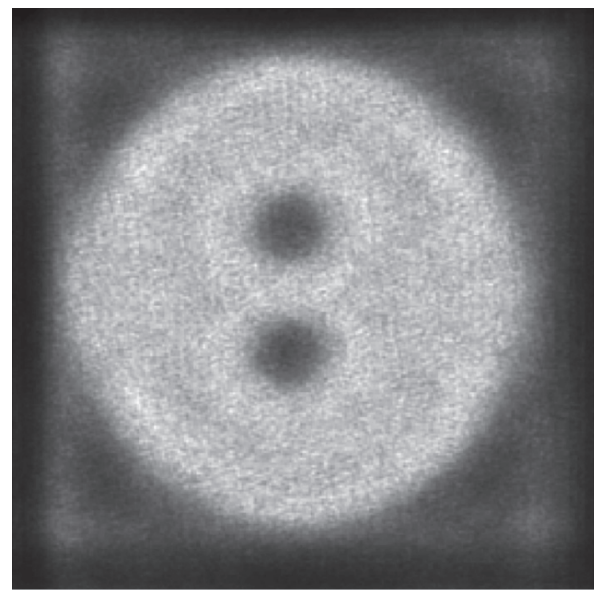

(c)

FIgURE 6: Reconstructed image. (a) CGLS at the 12th iteration. (b) LSQR at the 29th iteration. (c) MRNSD at the 39th iteration.

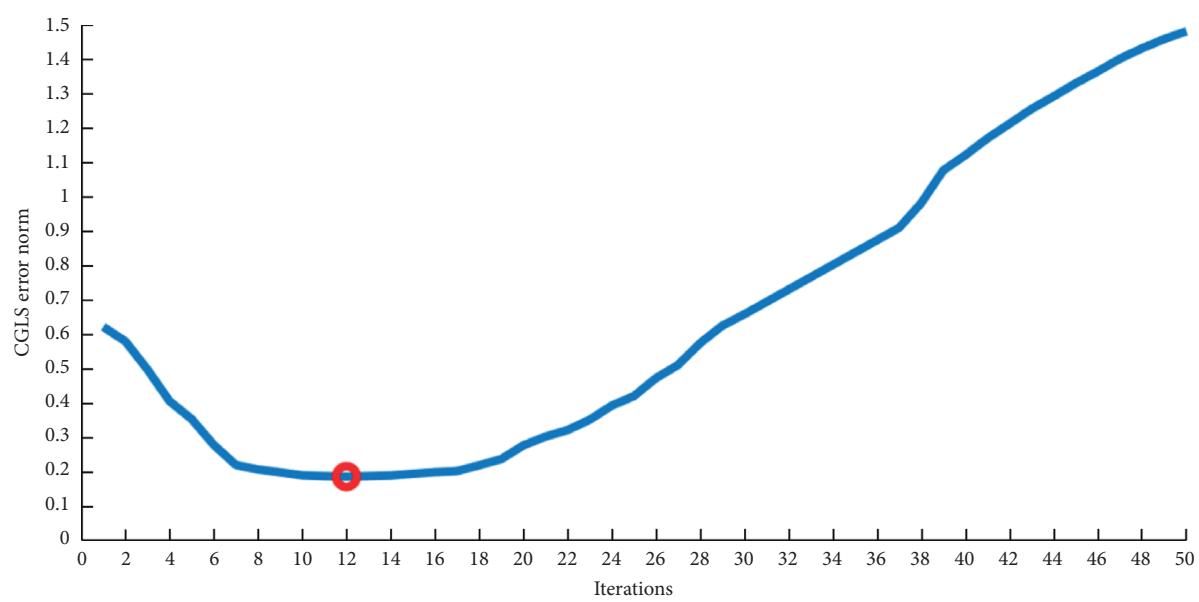

FIGURE 7: CGLS error norm versus the 50 first iterations.

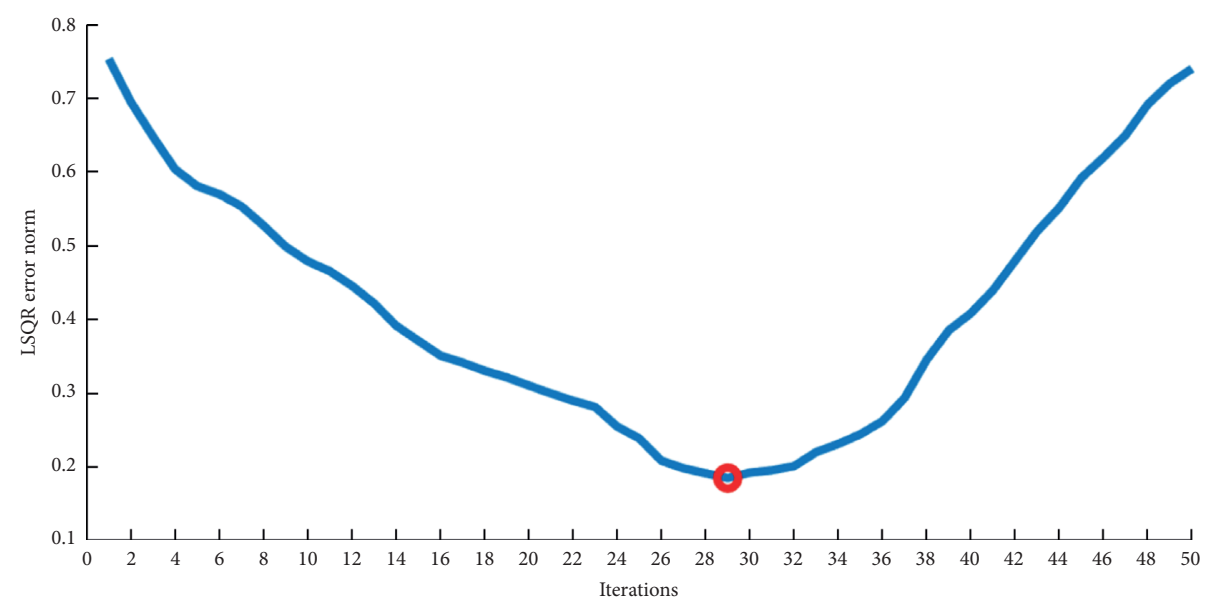

FIGURE 8: LSQR error norm versus the 50 first iterations. 
TABLE 1: History of error norm on various iterations.

\begin{tabular}{lccc}
\hline Iteration no. & & Error norm & \\
& CGLS & LSQR & 0.597240 \\
10 & 0.189991 & 0.479320 & 0.577751 \\
11 & 0.187661 & 0.465973 & 0.555261 \\
12 & $0.186189^{*}$ & 0.446252 & 0.529772 \\
13 & 0.187713 & 0.422392 & 0.509283 \\
14 & 0.189511 & 0.392066 & 0.274235 \\
27 & 0.510406 & 0.198770 & 0.255434 \\
28 & 0.573722 & 0.192086 & 0.244295 \\
29 & 0.624306 & $0.185546^{*}$ & 0.237046 \\
30 & 0.657604 & 0.193015 & 0.229797 \\
31 & 0.693474 & 0.196067 & 0.178303 \\
37 & 0.908695 & 0.294121 & 0.171797 \\
38 & 0.980268 & 0.345960 & $0.167680^{*}$ \\
39 & 1.075620 & 0.385787 & 0.169063 \\
40 & 1.119747 & 0.408994 & 0.169395 \\
41 & 1.168762 & 0.440049 & \\
\hline
\end{tabular}

${ }^{*}$ The optimal termination iteration for the given algorithm.

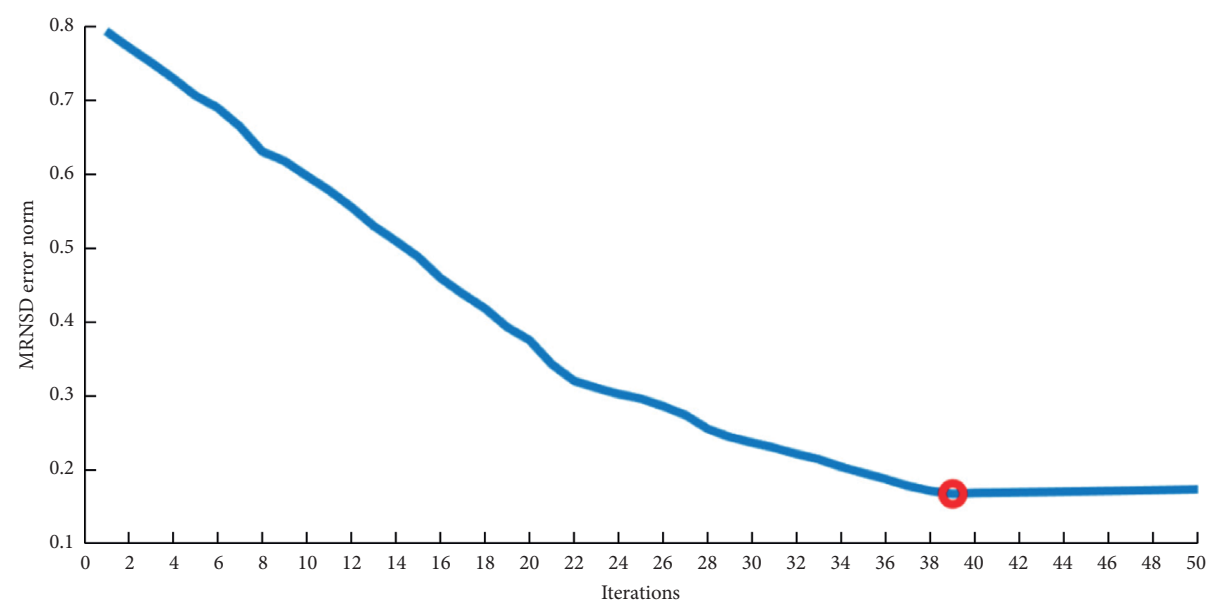

FIGURE 9: MRNSD error norm versus the 50 first iterations.

\section{Conclusions}

The least-squares method can be used to determine accurate, unbiased estimations of the parameters for a linear regression model. The conjugate gradient method is an efficient way of solving the related normal equations scheme [41]. In this article, when applying normal equations of least-quadratic problems to calculate the output of the estimators related to a linear pattern, we concentrate on reliable stopping criteria for a conjugate gradient algorithm. But, for other Krylov processes [11], such as LSQR, identical stop requirements can be formulated. In order to create stopping criteria, which, given an a priori probability, stop the conjugate gradient process, we will use the stochastic qualities of the linear regression pattern if stochastic variables can be considered with lesser probability of iteration running algorithm termination $[42,43]$.

If the conjugate gradient methods were used to solve the test problem, it would be very natural to apply stopping criteria that would take advantage of the minimization property of this type of method and also the stochastic properties of the underlying problem [11]. This is focused on the recent successful research in this area. The stopping criteria in this work are essential because of semiconvergence nature of the algorithms [10].

Three iterative approaches, CGLS, LSQR, and MRNSD algorithms, have been suggested in this paper. The proposed algorithms will filter the residual vector iteratively at each iteration to resolve the semiconvergence in CGLS, LSQR, and MRNSD. The problem tested in this work was a nonsquare problem. Furthermore, these algorithms can be problem tests on square problems for the future.

\section{Data Availability}

The code and data are available and will be provided upon request to the corresponding author.

\section{Conflicts of Interest}

The authors declare that they have no conflicts of interest. 


\section{Authors' Contributions}

Nasif R. Jaffri and Ahmad Almogren conceptualized the study and wrote the original draft; Ahmad Almogren acquired funding; Nasif R. Jaffri, Ayman Radwan, and Usama Abrar investigated the study; Aftab Ahmad was responsible for project administration; Aftab Ahmad and Farzan A. Khan wrote, reviewed, and edited the manuscript; Nasif R. Jaffri, Ahmad Almogren, and Ayman Radwan revised the article.

\section{Acknowledgments}

This work was supported by King Saud University, Riyadh, Saudi Arabia, through Researchers Supporting Project number RSP-2021/184. The work of author Ayman Radwan was supported by FCT/MEC through Programa Operacional Regional do Centro and by the European Union through the European Social Fund (ESF) under Investigador FCT Grant (5G-AHEAD IF/FCT- IF/01393/2015/CP1310/CT0002).

\section{References}

[1] A. F. Velo, D. V. S. Carvalho, and M. M. Hamada, "Liquid distribution and holdup in the random packed column," Flow Measurement and Instrumentation, vol. 62, pp. 176-185, 2018.

[2] R. Kotzé, A. Adler, A. Sutherland, and C. N. Deba, "Evaluation of electrical resistance tomography imaging algorithms to monitor settling slurry pipe flow," Flow Measurement and Instrumentation, vol. 68, Article ID 101572, 2019.

[3] J. Sun and W. Yang, "A dual-modality electrical tomography sensor for measurement of gas-oil-water stratified flows," Measurement, vol. 66, pp. 150-160, 2015.

[4] A. Magnusson, R. Rundqvist, A. E. Almstedt, and F. Johnsson, "Dual fibre optical probe measurements of solids volume fraction in a circulating fluidized bed," Powder Technology, vol. 151, no. 1-3, pp. 19-26, 2005.

[5] B. Ismail and W. Ahmed, "Innovative techniques for twophase flow measurements," Recent Patents on Electrical Engineering, vol. 1, pp. 1-13, 2010.

[6] K. S. Caldemeyer and K. A. Buckwalter, "The basic principles of computed tomography and magnetic resonance imaging," Journal of the American Academy of Dermatology, vol. 41, no. 5, pp. 768-771, 1999.

[7] E. Niemi, M. Lassas, A. Kallonen, L. Harhanen, K. Hämäläinen, and S. Siltanen, "Dynamic multi-source X-ray tomography using a spacetime level set method," Journal of Computational Physics, vol. 291, pp. 218-237, 2015.

[8] X. Li, A. J. Jaworski, and X. Mao, "Comparative study of two non-intrusive measurement methods for bubbling gas-solids fluidized beds: electrical capacitance tomography and pressure fluctuations," Flow Measurement and Instrumentation, vol. 62, pp. 255-268, 2018.

[9] H. Villarraga-Gómez, E. L. Herazo, and S. T. Smith, "Progression of X-ray computed tomography from medical imaging to current status in dimensional metrology," Precision Engineering, vol. 60, pp. 544-569, 2019.

[10] J. Huang, T. Z. Huang, X. L. Zhao, Z. B. Xu, and X. G. Lv, "Two soft-thresholding based iterative algorithms for image deblurring," Information Sciences, vol. 271, pp. 179-195, 2014.

[11] Y. L. Lai, W. W. Lin, and D. Pierce, "Conjugate gradient and minimal residual methods for solving symmetric indefinite systems," Journal of Computational and Applied Mathematics, vol. 84, no. 2, pp. 243-256, 1997.

[12] B. Qiao, X. Zhang, J. Gao, R. Liu, and X. Chen, "Sparse deconvolution for the large-scale Ill-posed inverse problem of impact force reconstruction," Mechanical Systems and Signal Processing, vol. 83, pp. 93-115, 2017.

[13] G. Guo, G. Tong, L. Lu, and S. Liu, "Iterative reconstruction algorithm for the inverse problems in electrical capacitance tomography," Flow Measurement and Instrumentation, vol. 64, pp. 204-212, 2018.

[14] J. Huang, H. Feng, Z. Xu, Q. Li, and Y. Chen, “A robust deblurring algorithm for noisy images with just noticeable blur," Optik, vol. 168, pp. 577-589, 2018.

[15] P. Jidesh and B. Banothu, "Image despeckling with non-local total bounded variation regularization," Computers \& Electrical Engineering, vol. 70, pp. 631-646, 2018.

[16] M. Bellalij, L. Reichel, and H. Sadok, "Some properties of range restricted GMRES methods," Journal of Computational and Applied Mathematics, vol. 290, pp. 310-318, 2015.

[17] Q. Zhong, C. Wu, Q. Shu, and R. W. Liu, "Spatially adaptive total generalized variation-regularized image deblurring with impulse noise," Journal of Electronic Imaging, vol. 27, no. 5, p. 1, 2018.

[18] C. J. Budd, M. A. Freitag, and N. K. Nichols, "Regularization techniques for Ill-posed inverse problems in data assimilation," Computers \& Fluids, vol. 46, no. 1, pp. 168-173, 2011.

[19] L. Jing, S. Liu, L. Zhihong, and S. Meng, "An image reconstruction algorithm based on the extended tikhonov regularization method for electrical capacitance tomography," Measurement, vol. 42, no. 3, pp. 368-376, 2009.

[20] T. Li, H. Chen, M. Zhang, S. Liu et al., "A new design in iterative image deblurring for improved robustness and performance," Pattern Recognition, vol. 90, pp. 134-146, 2019.

[21] V. Sizikov and D. Sidorov, "Generalized quadrature for solving singular integral equations of abel type in application to infrared tomography," Applied Numerical Mathematics, vol. 106, pp. 69-78, 2016.

[22] H. Zareiforoush, S. Minaei, M. R. Alizadeh, and A. Banakar, "A hybrid intelligent approach based on computer vision and fuzzy logic for quality measurement of milled rice," Measurement, vol. 66, pp. 26-34, 2015.

[23] Y. Chen, Y. Zhang, K. Zhang, Y. Deng et al., "FIRT: filtered iterative reconstruction technique with information restoration," Journal of Structural Biology, vol. 195, no. 1, pp. 49-61, 2016.

[24] Y. Xu, Y. Pei, and F. Dong, "An adaptive tikhonov regularization parameter choice method for electrical resistance tomography," Flow Measurement and Instrumentation, vol. 50, pp. 1-12, 2016.

[25] M. E. Hochstenbach, N. McNinch, and L. Reichel, "Discrete ill-posed least-squares problems with a solution norm constraint," Linear Algebra and its Applications, vol. 436, no. 10, pp. 3801-3818, 2012.

[26] S. Ren, J. Zhao, and F. Dong, "Dimensionality reduced simultaneous iterative reconstruction technique for electrical resistance tomography," Flow Measurement and Instrumentation, vol. 46, pp. 284-291, 2015.

[27] A. H. Bentbib, M. El Guide, K. Jbilou, and L. Reichel, "Global golub-kahan bidiagonalization applied to large discrete illposed problems," Journal of Computational and Applied Mathematics, vol. 322, pp. 46-56, 2017.

[28] I. Hnětynková, M. Kubínová, and M. Plešinger, "Noise representation in residuals of LSQR, LSMR, and CRAIG 
regularization," Linear Algebra and Its Applications, vol. 533, pp. 357-379, 2017.

[29] C. S. Liu, "Modifications of steepest descent method and conjugate gradient method against noise for ill-posed linear systems," Communications in Numerical Analysis, vol. 2012, Article ID cna-00115, 24 pages, 2012.

[30] J. W. Buurlage, R. H. Bisseling, and K. J. Batenburg, "A geometric partitioning method for distributed tomographic reconstruction," Parallel Computing, vol. 81, pp. 104-121, 2019.

[31] J. Kryszyn, P. Wróblewski, M. Stosio, D. Wanta, T. Olszewski, and W. T. Smolik, "Architecture of EVT4 data acquisition system for electrical capacitance tomography," Measurement, vol. 101, pp. 28-39, 2017.

[32] D. Barron, "Basic science: computed tomography," Current Orthopaedics, vol. 19, no. 1, pp. 20-26, 2005.

[33] U. Abrar, L. Shi, N. R. Jaffri, M. Short, and K. Hasham, "Electrical and mechanical sensor-based mass flow rate measurement system: a comparative approach," in Proceedings of the 2020 4th International Conference on Robotics and Automation Sciences, ICRAS, Wuhan, China, June 2020.

[34] W. Zhang, C. Wang, W. Yang, and C. H. Wang, "Application of electrical capacitance tomography in particulate process measurement-a review," Advanced Powder Technology, vol. 25, no. 1, pp. 174-188, 2014.

[35] Y. Jia, V. Chernyshev, and M. Skliar, "Ultrasound measurements of segmental temperature distribution in solids: method and its high-temperature validation," Ultrasonics, vol. 66, pp. 91-102, 2016.

[36] J. Song, Y. Hong, G. Wang, and H. Pan, "Algebraic tomographic reconstruction of two-dimensional gas temperature based on tunable diode laser absorption spectroscopy," Applied Physics B, vol. 112, no. 4, pp. 529-537, 2013.

[37] T. Wondrak, J. Pal, F. Stefani, V. Galindo, and S. Eckert, "Visualization of the global flow structure in a modified Rayleigh-bénard setup using contactless inductive flow tomography," Flow Measurement and Instrumentation, vol. 62, pp. 269-280, 2018.

[38] S. Borgwardt and C. Viss, "An implementation of steepestdescent augmentation for linear programs," Operations Research Letters, vol. 48, no. 3, pp. 323-328, 2020.

[39] A. E. Kostopoulos and T. N. Grapsa, "Self-scaled conjugate gradient training algorithms," Neurocomputing, vol. 72, no. 13-15, pp. 3000-3019, 2009.

[40] N. R. Jaffri, L. Shi, and U. Abrar, "Solving least square problem in tomography," in Proceedings of the Proceedings of the 2020 The 4th International Conference on Graphics and Signal Processing, pp. 56-60, ACM, New York, NY, USA, June 2020.

[41] P. Xiong, J. Deng, T. Lu, Q. Lu, Y. Liu, and Y. Zhang, “A sequential conjugate gradient method to estimate heat flux for nonlinear inverse heat conduction problem," Annals of $\mathrm{Nu}$ clear Energy, vol. 149, Article ID 107798, 2020.

[42] G. Landi, E. Loli Piccolomini, and I. Tomba, "A stopping criterion for iterative regularization methods," Applied Numerical Mathematics, vol. 106, pp. 53-68, 2016.

[43] M. Hanke and J. Nagy, "Inverse toeplitz preconditioners for ill-posed problems," Linear Algebra and its Applications, vol. 284, no. 1-3, pp. 137-156, 1998. 